\title{
Cleveland Multiport Catheter
}

National Cancer Institute

\section{Source}

National Cancer Institute. Cleveland Multiport Catheter. NCI Thesaurus. Code C159858.

A catheter designed for convection-enhanced delivery to the brain, incorporating four microcatheters. 